\title{
Automatic analysis of altered gait in arylsulphatase A-deficient mice in the open field
}

\author{
Toon Leroy, Stijn Stroobants, Jean-Marie Aerts, \\ Rudi D'Hooge, ANd DANiEl BerckMans \\ Katholieke Universiteit Leuven, Leuven, Belgium
}

\begin{abstract}
In current research with laboratory animals, observing their dynamic behavior or locomotion is a laborintensive task. Automatic continuous monitoring can provide quantitative data on each animal's condition and coordination ability. The objective of the present work is to develop an automated mouse observation system integrated with a conventional open-field test for motor function evaluation. Data were acquired from 86 mice having a targeted disruption of the arylsulphatase A (ASA) gene and having lowered coordinated locomotion abilities as a symptom. The mice used were 36 heterozygotes ( 12 females) and 50 knockout mice ( 30 females) at the age of 6 months. The mice were placed one at a time into the test setup, which consisted of a Plexiglas cage $(53 \times 34.5 \times 26 \mathrm{~cm})$ and two fluorescent bulbs for proper illumination. The transparent cage allowed images to be captured from underneath the cage, so image information could be obtained about the dynamic variation of the positions of the limbs of the mice for gait reconstruction. Every mouse was recorded for 10 min. Background subtraction and color filtering were used to measure and calculate image features, which are variables that contain crucial information, such as the mouse's position, orientation, body outline, and possible locations for the mouse's paws. A set of heuristic rules was used to prune implausible paw features and label the remaining ones as front/hind and left/right. After we had pruned the implausible paw features, the paw features that were consistent over subsequent images were matched to footprints. Finally, from the measured footprint sequence, eight parameters were calculated in order to quantify the gait of the mouse. This automatic observation technique can be integrated with a regular open-field test, where the trajectory and motor function of a free-moving mouse are measured simultaneously.
\end{abstract}

In contemporary mouse phenomics (Gerlai, 2002), open-field locomotion and gait/footprint analysis have been proposed as essential protocols for the comprehensive behavioral phenotyping of mutant mice (Crawley, 2003; Crawley \& Paylor, 1997). Since its introduction in the 1930s by Calvin Hall, the open-field test has become one of the most widely used behavioral observation procedures in laboratory rats and mice (Walsh \& Cummins, 1976). The availability of video tracking equipment and data extraction procedures has contributed tremendously to its present popularity in many fields of behavioral neuroscience and allows automated measurement of now commonly accepted elements of rodent behavior (Wolfer, Madani, Valenti, \& Lipp, 2001).

Automated analysis tools - both commercial and experimental - for open-field tests have been available for some time. The Ethovision system provides fully automated real-time tracking data of the positions of multiple mice when they are distinguishably marked (Noldus, Spink, \& Tegelenbosch, 2001). Attempts to track the full posture of a single mouse required more complex modelbased techniques (Twining, Taylor, \& Courtney, 2001); the precision of feature extraction proved insufficient for the reliable extraction and classification of posture.

Gait analysis procedures have been included for the assessment of possible motor incoordination, ataxia, or peripheral dysfunctions. Manual methods for obtaining footprint patterns in laboratory mice initially used grease or Vaseline on white paper (Rushton, Steinberg, \& Tinson, 1963); these were later replaced by photographic paper and photo-developer (de Medinaceli, Freed, \& Wyatt, 1982). Neither of these methods was very practical. A cheaper and less toxic alternative using ink and paper remains the most frequently used procedure to date (Johnston, Zachary, Dellon, Seiler, \& Teplica, 1991; Jolicœur, Rondeau, Hamel, Butterworth, \& Barbeau, 1979).

Various attempts have been made to develop automated procedures, but despite the continuing need for concise and innovative rodent assays (Wahlsten, Rustay, Metten, $\&$ Crabbe, 2003), such procedures did not actually replace the often tedious manual methods (Crawley, 2003). To observe toe spread in rats, Walker, Evans, Meade, Resig, and Sisken (1994) introduced a method that uses a frosted Perspex runway to increase contrast when the rat's feet

D. Berckmans, daniel.berckmans@biw.kuleuven.be 
touch the surface and a ventrally placed camera to record the footprints. A similar technique was applied to measure stride time, swing, and stance (Clarke \& Still, 2001). In another system, rats were videotaped while walking on a runway, with two mirrors placed alongside the runway to obtain lateral and ventral split images (Dijkstra, Meek, Robinson, \& Gramsbergen, 2000). In a sophisticated but more expensive method facetiously called the CatWalk, animals walk on a glass floor through which light is shone from the long end (Hamers, Lankhorst, van Laar, Veldhuis, \& Gispen, 2001). When their paws touch the floor, light is deflected, such that only the contact area is visible. A drawback of this technique is that it requires interactive manual labeling or scoring of the prints, but many different variables can be recorded once the prints are labeled.

This article describes the continued development of a relatively simple automated gait analysis system that can be integrated with a conventional open-field setup with minor technical alterations (Leroy, Silva, D'Hooge, Aerts, \& Berckmans, 2009). Combining open-field and gait/footprint analysis heeds recommendations to enhance scalability and information density of rodent testing procedures (Gerlai, 2002). The system uses readily available equipment (e.g., regular lights instead of special refractive lighting described in other work), and with the proposed materials, lighting conditions that merely require a low-cost USB webcam for image acquisition are achieved.

\section{METHOD}

\section{Subjects}

The test subjects were 86 mice at the age of 6 months having a targeted disruption of the arylsulphatase A (ASA) gene and documented locomotion disabilities (Hess et al., 1996). The mice were subdivided into groups of 36 heterozygotes (12 females) and 50 knockout mice (30 females).

\section{Apparatus and Procedure}

A steel frame was custom built to fit a cage (length $=53 \mathrm{~cm}$, width $=35 \mathrm{~cm}$, height $=26 \mathrm{~cm}$ ) made from transparent plastic, which housed the mouse during experiments (see Figure 1). Also attached to the frame were two fluorescent bulbs at a distance of $23 \mathrm{~cm}$ from the cage at opposite sides for proper illumination. The transparent cage allowed for images to be captured by a USB webcam (Philips Toucam Pro) mounted at a distance of $80 \mathrm{~cm}$ underneath the cage and with its lens pointing upward. The webcam captured images with a resolution of $640 \times 480$ pixels at a frequency of $15 \mathrm{~Hz}$. From the camera's bottom-up view, the position of each of the paws could be observed in the camera image. After the first tests, two additional Plexiglas boards were placed inside the cage to further restrict the width to $18 \mathrm{~cm}$. This was done to ensure that the mouse would move closer to the central area of the camera image when crossing the cage.

Before the start of the experiments, a webcam image of the empty cage was taken for use as a background image in the image analysis procedure. The experiments consisted of a series of open-field tests on individual mice in the transparent cage in the test installation for a duration of 10 min each. During each experiment, video images were captured by a camera below the cage.

Camera calibration. The camera was oriented perpendicular to the cage floor, and the long sides of the cage were parallel to the image's horizontal axis. No images with calibration markers were made before the experiments. Therefore, radial lens distortion in the camera image could not be estimated but was assumed to be negligible. Under these constraints, the relation between image coordinates (in pixels) and coordinates on the cage floor (in centimeters) could be approximated by a simple scaling factor $f$. The scaling factor $f$ was determined by measuring the distance between opposite walls of the cage (53 and $35 \mathrm{~cm}$, respectively) along five equidistant lines in the horizontal direction and five in the vertical direction, giving distances of 620 and 410 pixels, respectively, with a maximal deviation of 1.5 pixels. The resulting calibration factor $f$ was calculated as $53 \mathrm{~cm} / 620$ pixels $=0.0855 \mathrm{~cm} /$ pixel. With a calibration error of 1.5 pixels $\times 0.0855 \mathrm{~cm} /$ pixel $=1.28 \mathrm{~mm}$, neglecting the radial distortion proved to be justified.

Image analysis. The image analysis algorithm used simple techniques to measure features of the mouse in each image automatically, taking advantage of the static camera setup. Also, several color features were measured in each image. By combining these features over subsequent images, the footprints of the mouse were identified and labeled in relation to their location within the body features as belonging to the left-front (LF), right-front (RF), left-hind (LH), or right-hind $(\mathrm{RH})$ paw. This procedure was repeated automatically for all of the images in each video recording, yielding a sequence of footprints that formed the gait pattern. The algorithm is represented schematically in Figure 2. After a sequence of footprints was acquired, eight standardized gait parameters based on the distances between the footprints were calculated.

Calculation of mouse features. With the background image of the empty cage taken prior to the experiments and the webcam in a fixed position during the experiments, the outline of the mouse could be estimated using background subtraction. The background image was subtracted from every image $\mathbf{I}(x, y, t)$ taken at time $t$ from the recorded video of the cage containing the mouse. Applying a threshold ( $10 \%$ of the image intensity's dynamic range) to the subtracted image resulted in an image $\mathbf{I}_{m}(x, y, t)$ that was the same size as the original image $\mathbf{I}(x, y, t)$, where

$$
\mathbf{I}_{m}(x, y, t)= \begin{cases}1 & \text { if }(x, y) \text { lies within mouse outline; } \\ 0 & \text { otherwise }\end{cases}
$$

The basic morphological operations' erosion and dilation (Soille, 2003) were singularly applied to this mask image to ensure that the mouse's tail was not included in the calculation of its features (see Figure 3). The location of the mouse $[x(t), y(t)]$ at time $t$ was calculated as the mass center of $\mathbf{I}_{m}$ :

$$
x(t)=\frac{\sum_{x, y} x \mathbf{I}_{m}(x, y, t)}{\sum_{x, y} \mathbf{I}_{m}(x, y, t)} y(t)=\frac{\sum_{x, y} y \mathbf{I}_{m}(x, y, t)}{\sum_{x, y} \mathbf{I}_{m}(x, y, t)} .
$$

The orientation $\theta(t)$, length $a(t)$, and width $b(t)$ of the mouse in the image were then estimated by fitting an ellipse shape (Figure 4), with its origin at $[x(t), y(t)]$, to the outline in $\mathbf{I}_{m}$.

Motion analysis. As "open-field test" implies, the mouse could move freely; therefore, only parts of every video recording were suitable for gait analysis. In fact, gait analysis was applied only to images in which the mouse was known to be running. In these images, the mouse's body had a stretched appearance, so that it sufficiently resembled the ellipse shape with length $a(t)$ and width $b(t)$, estimated during the calculation of the mouse's features.

In every video recording, running sequences were automatically separated from standing/resting sequences according to the mouse's speed $v(t)$. We estimated $v(t)$ from the trajectory of the mouse's locations $[x(t), y(t)]$ as

$$
v(t)=\frac{\sqrt{[x(t)-x(t-\Delta t)]^{2}+[y(t)-y(t-\Delta t)]^{2}}}{\Delta t},
$$

with $\Delta t=1 / 30$, the sample rate of the location $[x(t), y(t)]$. 


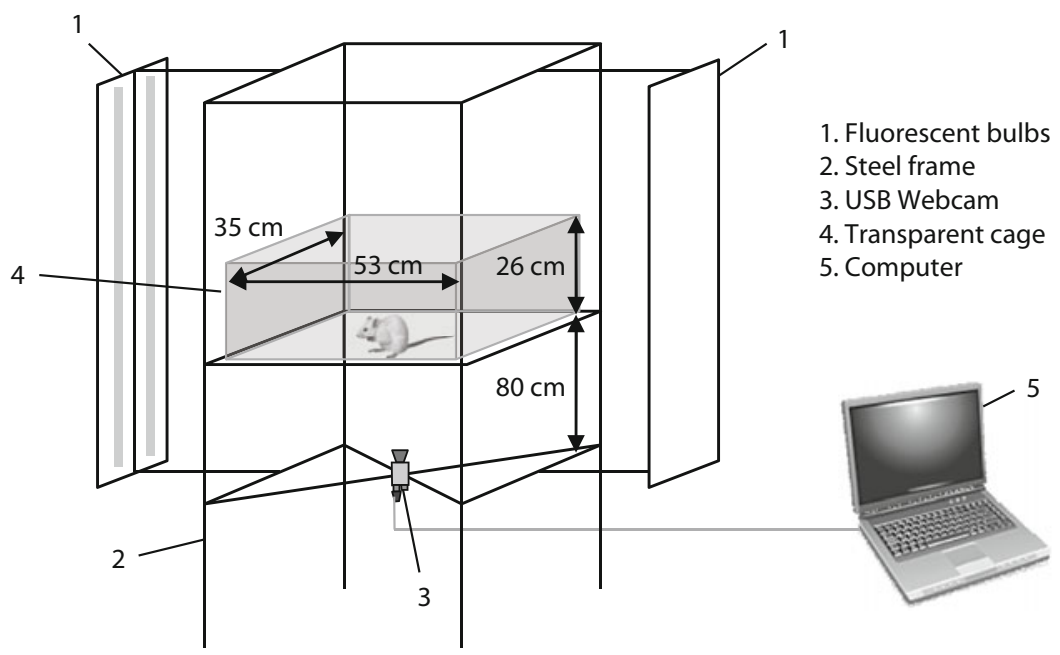

Figure 1. Schematic overview of the experimental setup.

Calculation of Mouse Features
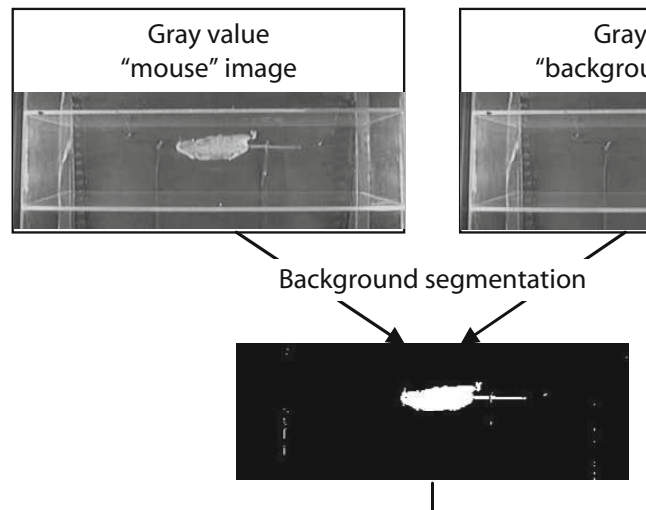

Morphological filtering

$\downarrow$

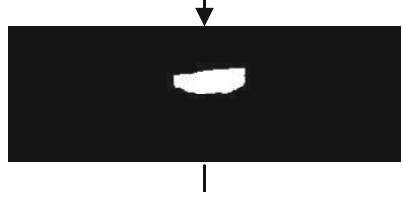

Location, orientation, and dimensions of mouse

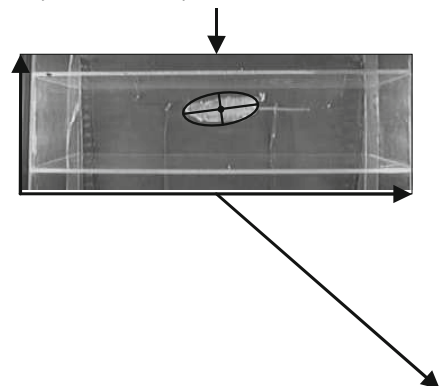

Gray value "background" image

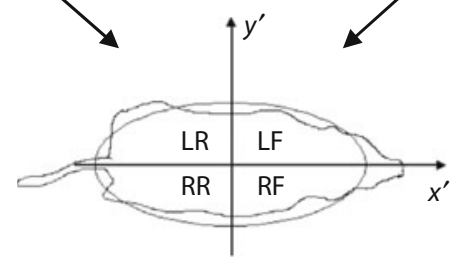

\section{Calculation of Paw Features}

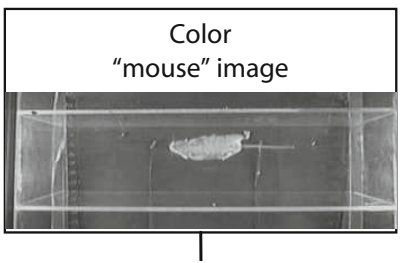

Color segmentation

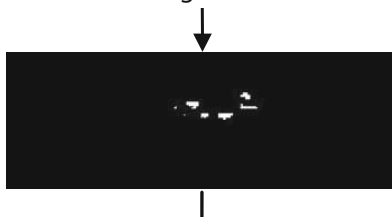

Morphological filtering

$\downarrow$

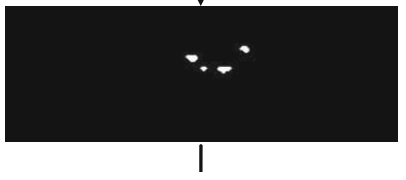

Location of paw objects

$\downarrow$

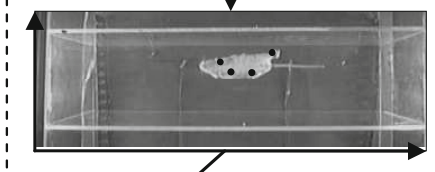

Figure 2. Schematic overview of the image analysis method used to measure the locations of the footprints and to identify them in the setup's bottom-up-view camera. 
A

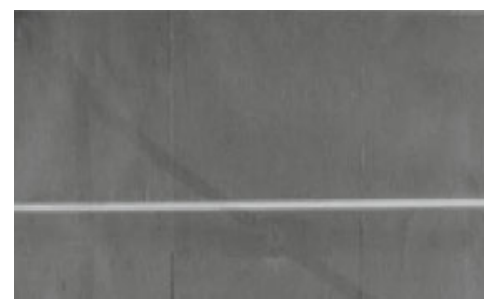

C

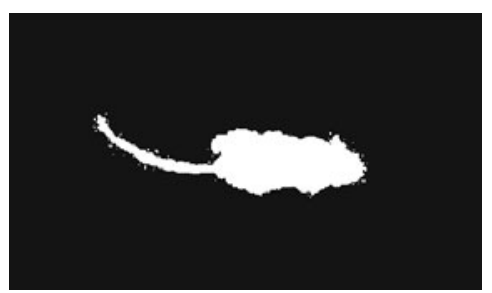

B

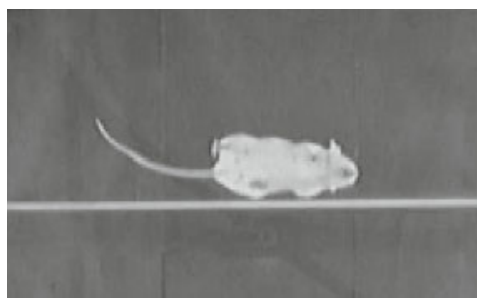

D

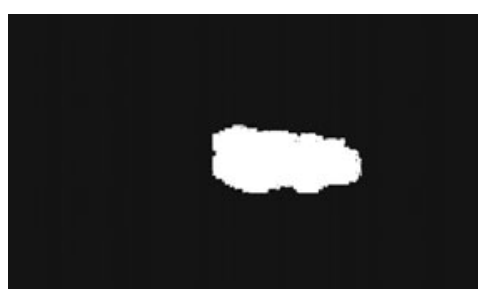

Figure 3. Illustration of the mouse detection algorithm. A background image (A) is subtracted from every incoming image $(B)$. The result $(C)$ is filtered to remove the tail and spurious noise from the recovered mouse outline (D). From "Automated Gait Analysis in the Open-Field Test for Laboratory Mice,” by T. Leroy, M. Silva, R. D'Hooge, J.-M. Aerts, and D. Berckmans, 2009, Behavior Research Methods, 41, p. 150. Copyright 2009 by The Psychonomic Society, Inc.

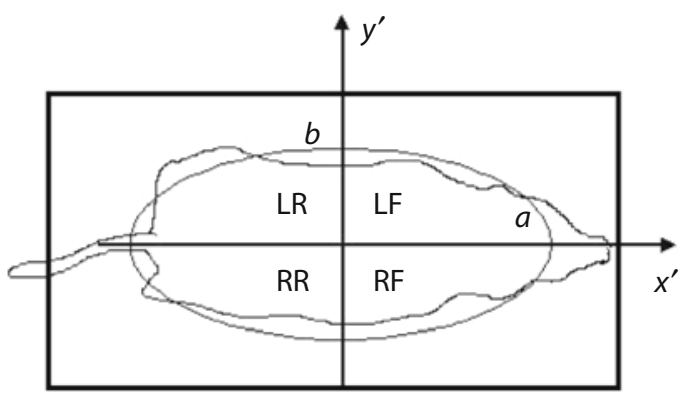

Figure 4. An ellipse shape fit to the mouse's outline. The bounding box has 1.5 times the dimensions of the ellipse. The bounding box was used to reject paw features lying outside and to identify the remaining paw features as left-front (LF), right-front (RF), left-hind ( $\mathrm{LH})$, or right-hind ( $\mathrm{RH})$, on the basis of their positions relative to the four quadrants. From "Automated Gait Analysis in the Open-Field Test for Laboratory Mice," by T. Leroy, M. Silva, R. D'Hooge, J.-M. Aerts, and D. Berckmans, 2009, Behavior Research Methods, 41, p. 150. Copyright 2009 by The Psychonomic Society, Inc.

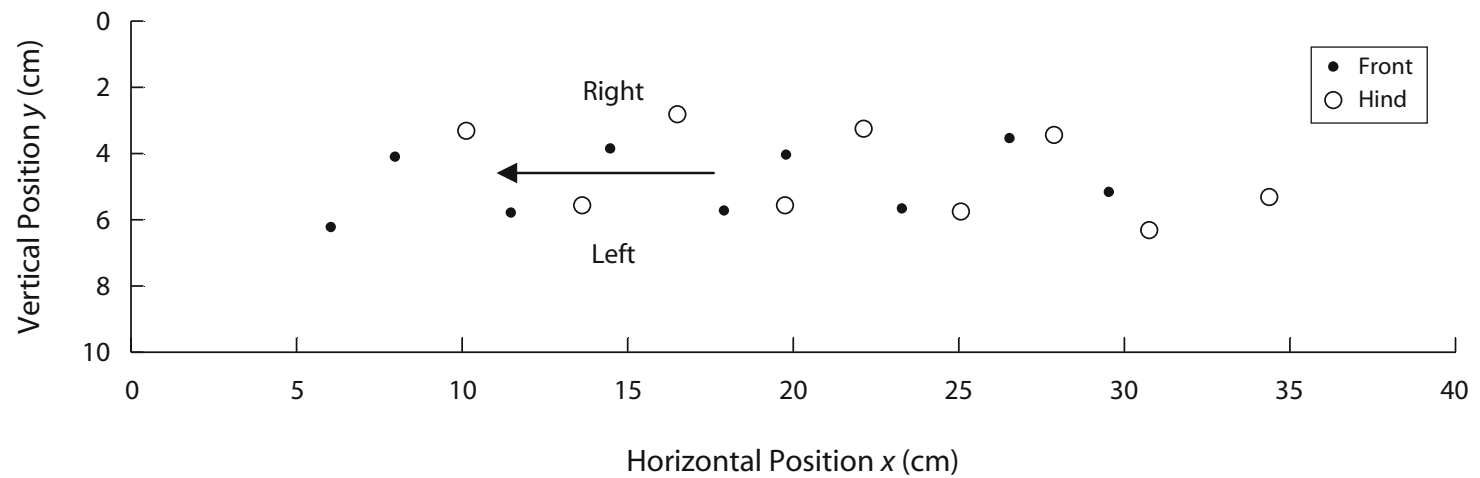

Figure 5. An example of a mouse's footprint pattern, as measured during a crossing from right to left. 
Table 1

Overview of the Eight Gait Parameters That Were Calculated From the Measured Footprint Sequence

\begin{tabular}{ll}
\hline \multicolumn{1}{c}{ Parameter } & \multicolumn{1}{c}{ Description } \\
\hline Right overlap (RO) & Distance between the positions of the front and hind limbs on the right side \\
Left overlap (LO) & Distance between the positions of the front and hind limbs on the left side \\
Front Base (FB) & Distance orthogonal to the walking direction between the front limbs \\
Hind base (HB) & Distance orthogonal to the walking direction between the hind limbs \\
Right front limb stride (RFS) & Distance between two sequential prints of the right front paws \\
Left front limb stride (LFS) & Distance between two sequential prints of the left front paws \\
Right hind limb stride (RHS) & Distance between two sequential prints of the right hind paws \\
Left hind limb stride (LHS) & Distance between two sequential prints of the left hind paws
\end{tabular}

A sequence of data with a minimal length of $1 \mathrm{sec}(=30 \mathrm{sub}-$ sequent images), where the speed $v(t)$ was above a threshold of $2 \mathrm{~cm} / \mathrm{sec}$, was regarded as a running sequence and was further processed for the detection of footprints.

Calculation of footprint features. To measure the location of the footprints, a color filter was applied to the camera image, within the region of the mouse outline. Let the three color bands of the RGB (red-green-blue) image $\mathbf{I}(x, y)$ be represented by $\mathbf{I}_{r}(x, y)$, $\mathbf{I}_{g}(x, y)$, and $\mathbf{I}_{b}(x, y)$. From these color bands, the following grayscale image was calculated, enhancing the pixels with a strong red color component:

$$
\mathbf{I}_{f}=(0.7) \mathbf{I}_{r}-(0.59) \mathbf{I}_{g}-(0.11) \mathbf{I}_{b} .
$$

This equation corresponds to the difference between the intensity of the red component and the total intensity of the image, the latter calculated as a weighed sum of the color components with coefficients $0.3,0.59$, and 0.11

Paw objects were detected by applying a threshold $(5 \%$ of the image intensity's dynamic range) to $\mathbf{I}_{f}$ and calculating the mass center $\left(x_{p i}, y_{p i}\right)$ of all $i=1 \ldots n$ unconnected objects in the resulting binary paw image $\mathbf{I}_{p}$. The number of paw objects $n$ varied between images and was often higher than the expected number of four paws. For instance, this was the case when the nose or tail of the mouse, being similar in color to the paws, passed the threshold. Also, a dirty paw could result in a footprint image that was fragmented into multiple unconnected objects. Therefore, the detected paw objects had to be pruned to eliminate the false positives. If paw objects $\left(x_{p i}, y_{p i}\right)$ were detected in three subsequent images within $2 \mathrm{~mm}$ of each other, this was recognized as a footprint with its location $\left(x_{f}, y_{f}\right)$ calculated as the mean of these paw objects' locations. This was successful for separating the paws that were in contact with the floor from the nose and tail, which were moving. It also averaged out the error in the measured position of the paw objects.

Finally, the recovered footprints were further pruned and identified as left-front (LF), right-front (RF), left-hind (LH), or righthind $(\mathrm{RH})$ by looking at their position relative to the ellipse that was formed by the mouse's features (see Figure 4).

To remove outliers from among the footprints, footprints that were located outside a bounding box with 1.5 times the length and width of the ellipse were removed. The remaining footprints were all located within different quadrants of the ellipse corresponding to the four paw labels (see Figure 4). For each footprint, the ellipse quadrant that contained it was determined and the corresponding label was assigned to that footprint.

This procedure was repeated automatically for all of the images within a video recording, resulting in a sequence of footprints, forming the gait pattern (Figure 5).

Gait parameters. From all sequences of footprint patterns - one measured from each video - eight gait parameters were calculated on the basis of the distances between footprints. These parameters - right overlap (RO), left overlap (LO), front base (FB), hind base (HB), right front limb stride (RFS), left front limb stride (LFS), right hind limb stride (RHS), and left hind limb stride (LHS) - are described in Table 1 and illustrated in Figure 6.

\section{RESULTS AND DISCUSSION}

No gait information could be obtained from 18 of the mice (5 heterozygotes, 13 knockouts) because of a lack of movement. These mice were excluded from the analysis.

\section{Measurement Accuracy}

The accuracy of footprint detection was evaluated by comparing a set of footprint locations from the automatic method with a corresponding reference data set. Reference data were created by manually locating the footprints in two selected videos using a custom software tool. One reference video contained a heterozygote mouse; the other contained a knockout mouse. In total, 203 footprints were manually labeled and were compared against their automatically measured counterparts, using the Euclidean distance as an error measure (see Table 2). Four footprints were found to be outliers; that is, the footprints were manually labeled but were not detected by the automatic method.

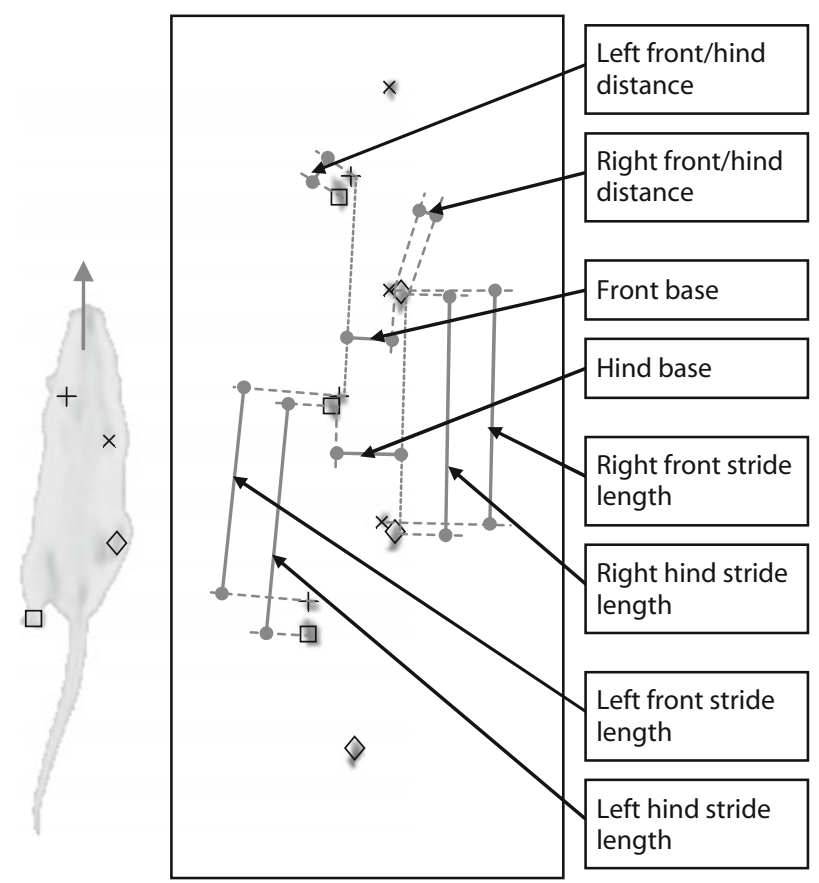

Figure 6. An overview of the eight gait parameters that were calculated from the footprint patterns. 
Table 2

Mean Standard Errors $\left(M S_{\mathrm{e}}\right)$ and Standard Deviations (SDs, in Millimeters) of the Automatic Method, As Compared With Manual Labeling

\begin{tabular}{lccccc}
\hline & \multicolumn{2}{c}{ Heterozygote } & & \multicolumn{2}{c}{ Knockout } \\
\cline { 2 - 3 } \cline { 5 - 6 } Footprint & $M S_{\mathrm{e}}$ & $S D$ & & $M S_{\mathrm{e}}$ & $S D$ \\
\hline Left front & 2.0 & 1.6 & 3.0 & 1.9 \\
Right front & 2.6 & 1.9 & 2.3 & 1.4 \\
Left hind & 4.2 & 1.6 & 3.3 & 1.5 \\
Right hind & 4.0 & 1.9 & 3.2 & 1.9 \\
\hline
\end{tabular}

On the basis of the above-mentioned measurements, the mean error was determined to be $3.1 \mathrm{~mm}(S D=1.8 \mathrm{~mm})$. In image coordinates, the mean error corresponds to less than 2 pixels $(S D=1$ pixel).

\section{A}

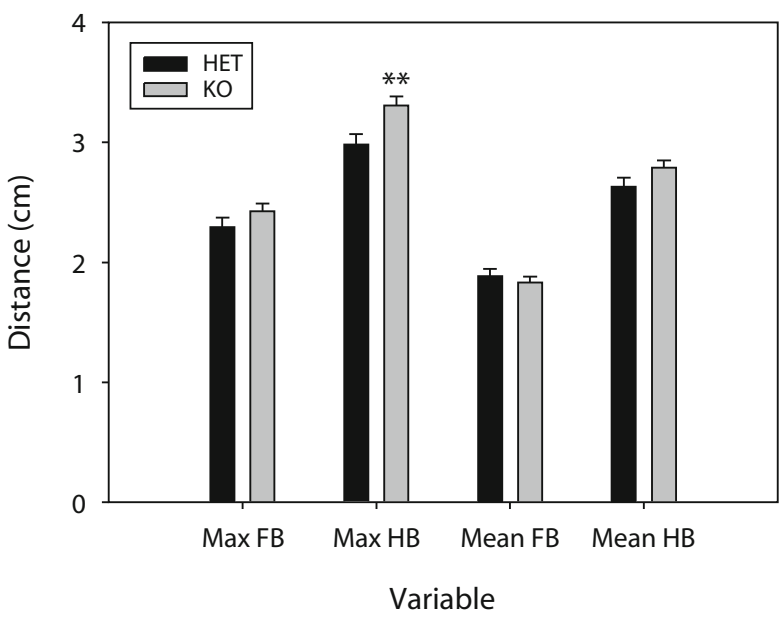

As can be seen from Table 2, the measurement error for the hind paws was larger than that for the front paws. A possible explanation is that the visible area of the paws is more elongated while in contact with the floor than just before or after, an effect that is more pronounced for the hind paws, which are larger than the front paws. The automatic method finds the location of the footprint as the average of the mass center over subsequent images, often including a location at which the paw is no longer fully in contact with the floor. Thus, again, this bias is larger for the hind paws than for the front paws.

\section{Gait Parameters}

The heterozygotes showed a gait pattern that was clearly distinctive from that of the knockout mice (see Figure 7).

\section{B}

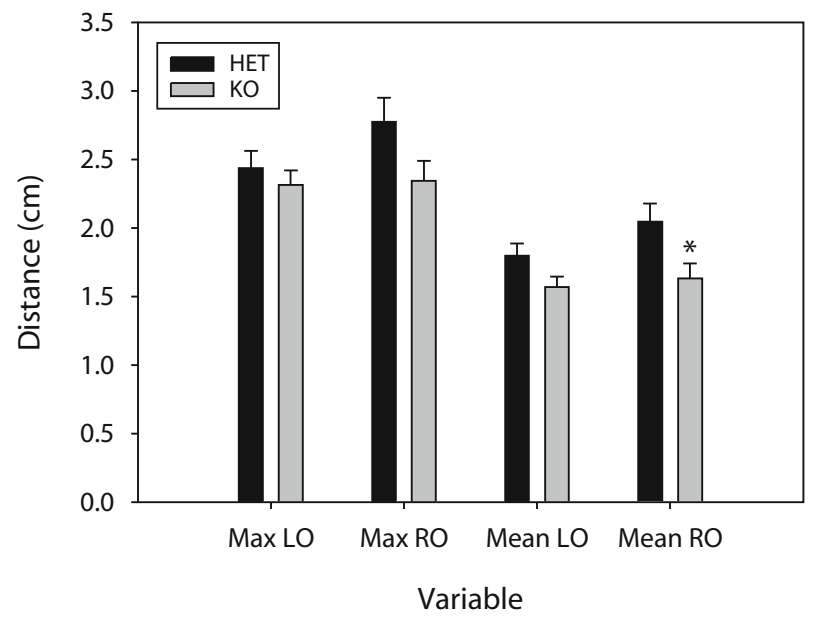

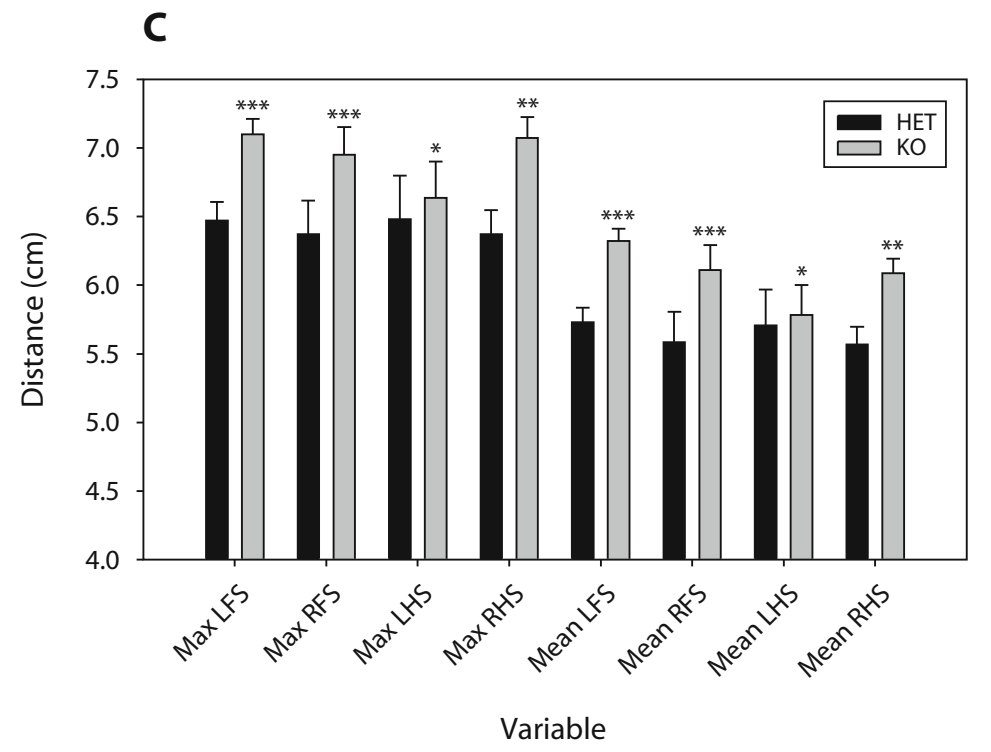

Figure 7. Gait parameters of heterozygotes (HET) and knockouts (KO) at the age of 6 months. (A) The mean and maximal front base (FB) and hind base (HB). (B) The mean and maximal left overlap (LO) and right overlap (RO). (C) The mean and maximal left front (LFS), right front (RFS), left hind (LHS), and right hind (RHS) stride lengths, respectively. ${ }^{* *} p<.05$. ${ }^{* *} p<.01$. ${ }^{* * *} p<{ }^{*}$ .001 . 

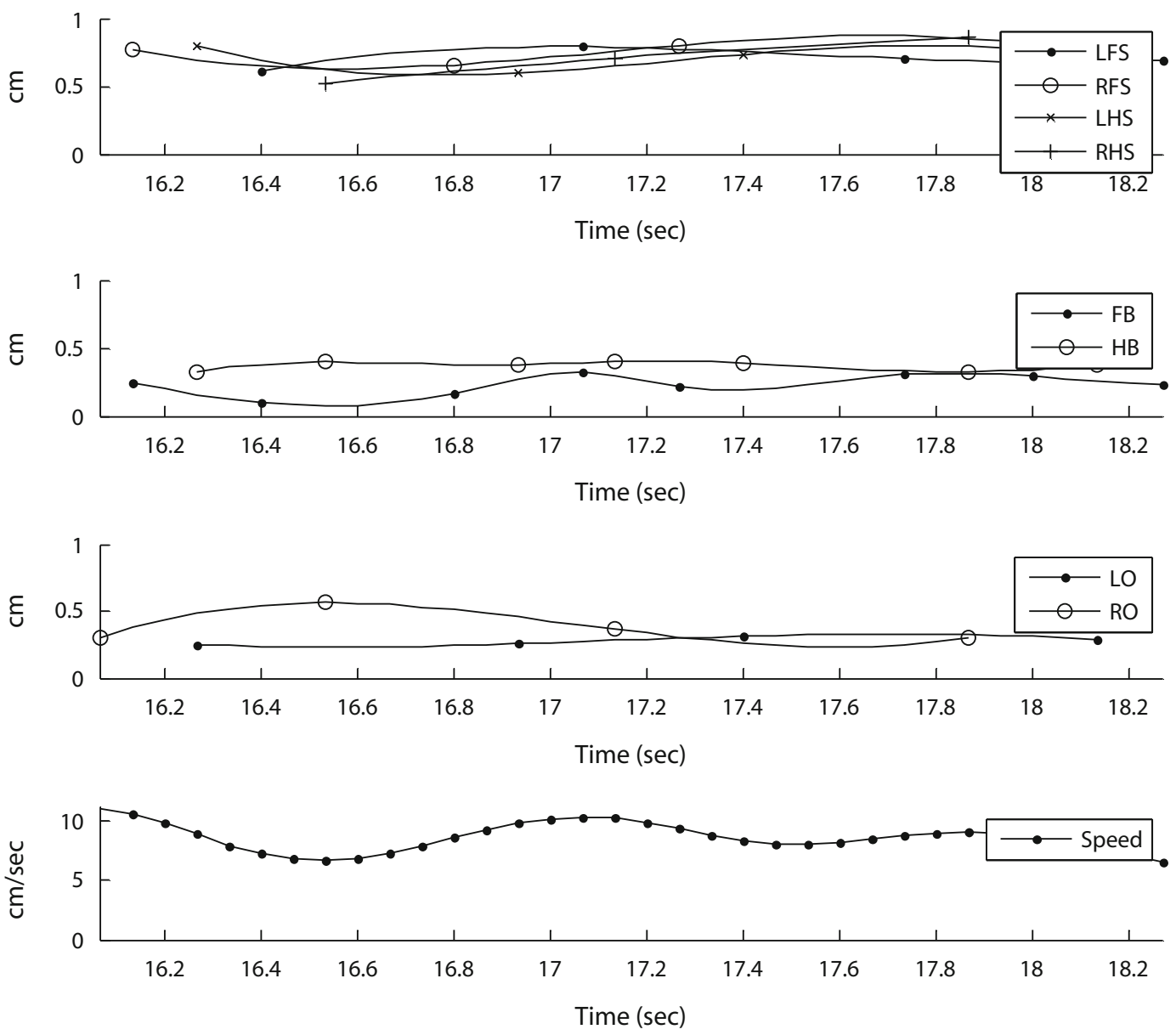

Figure 8. The gait parameters and run speed for one run sequence of a heterozygote, as a function of time. The gait parameters are the left front (LFS), right front (RFS), left hind (LHS), and right hind (RHS) stride lengths, front base (FB), hind base (HB), left overlap (LO), and right overlap (RO). The markers indicate when these parameters were measured. The smooth curve was added as a visual guide to illustrate the dynamic trends.

The knockout mice had longer stride lengths (LFS, RFS, LHS, RHS) for all paws $(p<.001$ for both front paws; $p<.01$ for the right hind paw; $p<.05$ for the left hind paw). The knockout mice had a higher maximum hind base $(p<.01)$. No difference was found between the genotypes with regard to the front base. The males displayed a larger mean $(p<.05)$ and maximum $(p<.01)$ FB.

Regarding overlap parameters, the heterozygotes displayed an increased mean right overlap $(p<.05)$ and a trend toward an elevated maximum $\mathrm{RO}(p=.067)$. In the left overlap, there was only a trend toward an increased mean $(p=.061)$. The males showed a larger mean $(p<$ $.05)$ and maximum $(p<.001)$ LO.

\section{Quantification of the Dynamic Nature of Mouse Gait}

It should be noted that, rather than being a constant process, the gait of a mouse is dynamic and time variant, depending on the speed and direction of the mouse's movements. This can be quantified and visualized by plotting the gait parameters of one run sequence of a heterozygote as a function of time, together with the speed (see Figure 8). The gait parameters used in the evaluation of the gait were obtained by averaging out this variation. This is also the reason why large numbers of mice and experiments were needed.

\section{CONCLUSION}

In this article, we introduced a novel method for gait analysis. This method can be combined with more classical methods of recording open-field behavior. We presented an automatic computer vision algorithm for detecting the mouse and the location of its paws in a captured image. Also, footprints were identified where the paws were in contact with the floor. From these footprints, eight parameters were quantified to characterize mouse gait. To validate this method, we examined normal heterozygotes and mutant mice with known gait defects. Analysis of gait parameters showed a distinctive difference in gait patterns between these groups. In this procedure, the subject may move freely in an open-field arena because the algorithm 
processes the paw positions within an axis relative to the location and orientation of the mouse.

\section{AUTHOR NOTE}

Portions of the present article were published previously in Behavior Research Methods, 41, 148-153. Correspondence concerning this article should be addressed to D. Berckmans, M3-BIORES, Katholieke Universiteit Leuven, Kasteelpark Arenberg 30, B-3001 Heverlee, Belgium (e-mail: daniel.berckmans@biw.kuleuven.be).

\section{REFERENCES}

Clarke, K. A., \& Still, J. (2001). Development and consistency of gait in the mouse. Physiology \& Behavior, 73, 159-164.

Crawley, J. N. (2003). Behavioral phenotyping of rodents. Comparative Medicine, 53, 140-146.

Crawley, J. N., \& Paylor, R. (1997). A proposed test battery and constellations of specific behavioral paradigms to investigate the behavioral phenotypes of transgenic and knockout mice. Hormones \& Behavior, 31, 197-211.

de Medinaceli, L., Freed, W. J., \& Wyatt, R. J. (1982). An index of the functional condition of rat sciatic nerve based on measurements made from walking tracks. Experimental Neurology, 77, 634-643.

Dijkstra, J. R., Meek, M. F., Robinson, P. H., \& Gramsbergen, A. (2000). Methods to evaluate functional nerve recovery in adult rats: Walking track analysis, video analysis and the withdrawal reflex. Journal of Neuroscience Methods, 96, 89-96.

GerLaI, R. (2002). Phenomics: Fiction or the future? Trends in Neurosciences, 25, 506-509.

Hamers, F. P. T., Lankhorst, A. J., van LaAR, T. J., Veldhuis, W. B., \& GISPEN, W. H. (2001). Automated quantitative gait analysis during overground locomotion in the rat: Its application to spinal cord contusion and transection injuries. Journal of Neurotrauma, 18, 187-201.

Hess, B., Saftig, P., Hartmann, D., Coenen, R., LüllmannRauch, R., Goebel, H. H., et AL. (1996). Phenotype of arylsulphatase A-deficient mice: Relationship to human metachromatic leukodystrophy. Proceedings of the National Academy of Sciences, 93, 14821-14826.
Johnston, R. B., Zachary, L., Dellon, A. L., Seiler, W. A., IV, \& TePLICA, D. M. (1991). Improved imaging of rat hindfoot prints for walking track analysis. Journal of Neuroscience Methods, 38, 111114.

Jolicceur, F. B., Rondeau, D. B., Hamel, E., Butterworth, R. F., \& BARBEAU, A. (1979). Measurement of ataxia and related neurological signs in the laboratory rat. Canadian Journal of Neurological Sciences, 6, 209-215.

Leroy, T., Silva, M., D’Hooge, R., Aerts, J.-M., \& Berckmans, D. (2009). Automated gait analysis in the open-field test for laboratory mice. Behavior Research Methods, 41, 148-153.

Noldus, L. P. J. J., Spink, A. J., \& Tegelenbosch, R. A. J. (2001). EthoVision: A versatile video tracking system for automation of behavioral experiments. Behavior Research Methods, Instruments, \& Computers, 33, 398-414.

Rushton, R., Steinberg, H., \& Tinson, C. (1963). Effects of a single experience on subsequent reactions to drugs. British Journal of Pharmacology \& Chemotherapy, 20, 99-105.

SoIlle, P. (2003). Morphological image analysis: Principles and applications (2nd ed.). New York: Springer.

Twining, C. J., TAYlor, C. J., \& CourTney, P. (2001). Robust tracking and posture description for laboratory rodents using active shape models. Behavior Research Methods, Instruments, \& Computers, 33, 381-391.

Wahlsten, D., Rustay, N. R., Metten, P., \& Crabbe, J. C. (2003). In search of a better mouse test. Trends in Neurosciences, 26, 132-136.

Walker, J. L., Evans, J. M., Meade, P., Resig, P., \& Sisken, B. F. (1994). Gait-stance duration as a measure of injury and recovery in the rat sciatic nerve model. Journal of Neuroscience Methods, 52, 47-52.

Walsh, R. N., \& Cummins, R. A. (1976). The open-field test: A critical review. Psychological Bulletin, 83, 482-504.

Wolfer, D. P., Madani, R., VAlenti, P., \& LiPP, H.-P. (2001). Extended analysis of path data from mutant mice using the public domain software Wintrack. Physiology \& Behavior, 73, 745-753.

(Manuscript received November 14, 2008; revision accepted for publication January 22, 2009.) 